\title{
Scattering and Annihilation Experiments Using a Trap-Based Beam
}

\author{
J. P. Sullivan', L. J. Barnes ${ }^{2}$, J. P. Marler², S. J. Gilbert ${ }^{2}$ and C. M. Surko² \\ ${ }^{1}$ Photon Factory, KEK, 1-1 Oho Tsukuba, Ibaraki 305-0801, Japan. email:sullivan@nabiki.kek.jp \\ ${ }^{2}$ Physics Department, University of California San Diego, 9500 Gilman Dr., La Jolla, 92093, Ca, \\ USA. email:csurko@ucsd.edu
}

\section{Keywords: Positron Scattering, Annihilation, Resonances, Low energy, Clusters}

\begin{abstract}
The buffer gas trap technique for positron trapping and beam formation has made available a low energy, high resolution positron beam for the first time. This has opened the way for a range of new studies in positron-atom(molecule) scattering, including measurements of annihilation cross sections $\left(\mathrm{Z}_{\mathrm{eff}}\right)$ as a function of energy and processes such as excitation by low energy positron impact. New light is now being shed on the interactions of positrons with ordinary matter, including the first observation of positron-molecule resonances, and the latest progress in these studies is summarised here. The application of these techniques to the studies of larger systems, such as clusters, is also briefly discussed.
\end{abstract}

\section{Introduction}

Detailed studies of low energy $(<50 \mathrm{eV})$ positron scattering processes have been hampered by the difficulty in obtaining a high resolution beam [1]. Typical positron beams for these studies were produced using a moderating material such as tungsten, which gives a resolution of $>0.5 \mathrm{eV}$ [2]. Studies of ionisation [3], positronium formation [4] and total scattering cross sections [5] have been made, along with some differential scattering measurements, but measurements at very low energy $(<5 \mathrm{eV})$ and of excitation processes have been sparse [1]. Recently, techniques have been developed to trap large numbers of positrons (up to $3 \times 10^{8}$ in under 10 minutes) and cool them to room temperature $(\sim 25 \mathrm{meV})$ using the combination of confining magnetic and electrostatic fields and a buffer gas [6]. This reservoir of positrons can then be used as a source for a positron beam with an energy spread as low as $18 \mathrm{meV}$ [7]. The beam is guided by a magnetic field of similar magnitude to the confining field, and any scattering process also takes place in the field. This has led to new experimental techniques and analysis that takes advantage of the behaviour of the positrons in such an environment [8]. Using these techniques, experiments have been performed for the first time that investigate processes such as positron annihilation as a function of energy and excitation of atomic and molecular targets [9]. The positron beam also has potential application to the study of clusters, providing both new and complementary information to studies using electron scattering.

\section{The Positron Trap and Beam}

Descriptions of the operation of the buffer gas trap and positron beam formation have been published in various references [6,7], only a brief overview is presented here. Positrons from a radioactive ${ }^{22} \mathrm{Na}$ source are moderated using a solid neon moderator maintained at $\sim 7 \mathrm{~K}$. The resultant low energy positrons are guided into a three stage buffer gas trap, shown in Fig. 1, using electrostatic and magnetic fields. Once inside the trap the positrons collide with the background $\mathrm{N}_{2}$, exciting an electronic transition of the molecule, thereby losing energy and becoming confined in the electrostatic well. Successive collisions lead to confinement in the third, lowest pressure, stage where the positrons cool to room temperature by exciting vibrational and rotational modes in the buffer gas. A small amount of $\mathrm{CF}_{4}$ is added to this stage to enhance the cooling. The efficiency of 
the trap can be as high as $25 \%$ (positrons trapped per moderated positrons entering). As many as $3 \times 10^{8}$ positrons have been accumulated using this method, in a period of less than 10 minutes [10].

By raising the bottom of the confining well, the trapped positrons are forced over a well defined potential barrier and form a beam. Careful application of this technique minimises the energy spread of the positrons. This results in a positron beam with an energy resolution of $25 \mathrm{meV}$ or less, with the resolution being limited by the space charge of the trapped positrons. The energy of the beam is tuneable from $50 \mathrm{meV}$ upwards, and is determined by the difference in potential between the barrier and the region though which the beam is travelling. The temporal spread of the beam is typically a few $\mu$ s with approximately 30,000 positrons per pulse at a repetition rate of $4 \mathrm{~Hz}$ [8]. This process is illustrated in Fig. 2. Positrons hitting the back wall of the chamber are detected via the annihilation gamma rays produced, using a $\mathrm{NaI}$ detector.

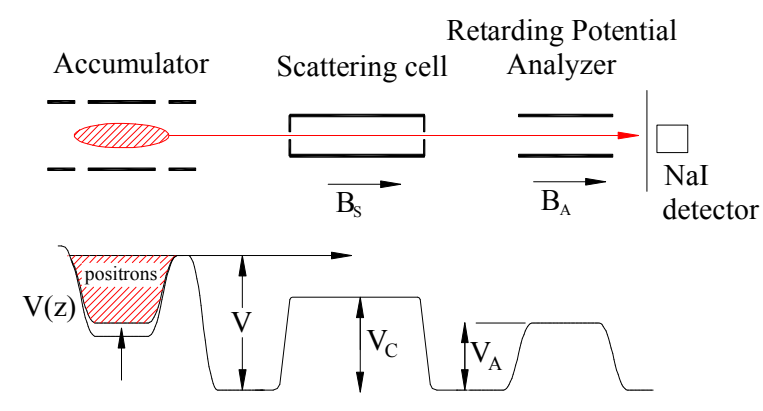

Figure 2: Schematic of the beam formation scheme, showing the setup for scattering measurements.

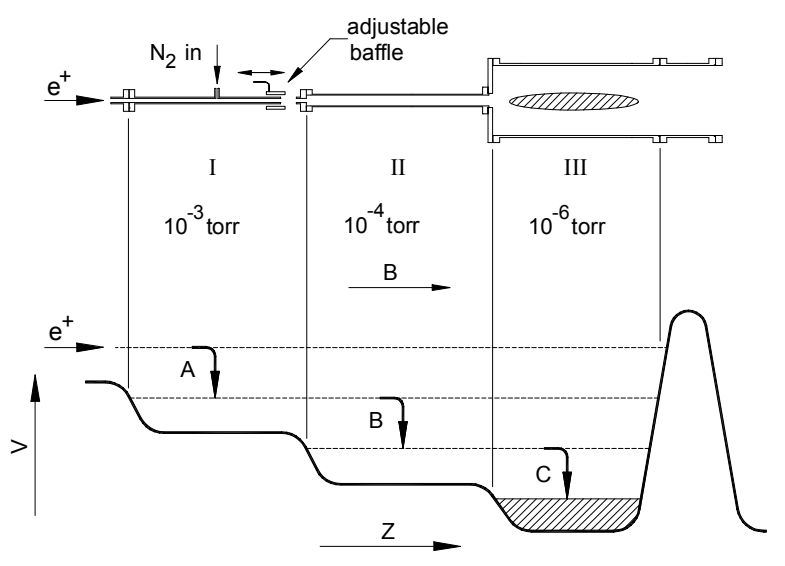

Figure 1: Schematic of the trapping process, showing the electrode structure and electrostatic potential well configuration.

Once formed, the beam can be directed through a gas cell containing the target gas of choice. Interactions with the gas will affect the energy distribution of the beam as well as result in 511 $\mathrm{keV}$ photons if annihilation takes place. By measuring the energy distribution of the positron beam after its transit through the gas cell, information about various scattering processes can be obtained. Measurement of the annihilation gamma rays yields information on the annihilation cross section. These processes will be discussed in more detail below.

\section{Scattering Measurements}

Annihilation. For annihilation measurements, the experimental setup shown in Fig. 3 was used [11]. The positron beam was directed through a gas cell containing the target species of choice. The potential (and thus the beam energy) was constant over the interaction region viewed by a CsI detector which is sensitive to $511 \mathrm{keV}$ gamma rays from the annihilation of a positron with an electron in the target. The positron beam is kept in flight using a potential barrier at the end of the apparatus, making up to 10 passes before the barrier is lowered and the beam annihilates. This allows signal rates to be enhanced relative to a single pass, as well as avoiding problems due to background

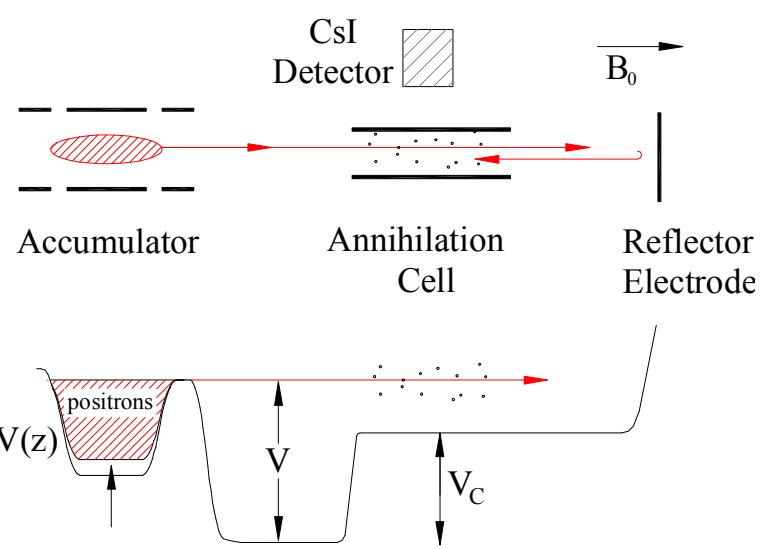

Figure 3: Schematic of the setup for annihilation measurements. 
from the beam annihilation. Gas pressure in the interaction region is typically 0.1 Torr to $1 \mu$ Torr. Signals can be as low as 1 count per $10^{7}$ positrons, with a background of 1 count per $10^{9}$ positrons. Absolute calibration of the annihilation cross section has an uncertainty of $25 \%$, with the relative uncertainty determined only by counting time.

This technique has been used to study hydrocarbon and substituted hydrocarbon molecules, which display very high annihilation rates [12]. The annihilation cross section is presented as $Z_{\text {eff, which }}$ is the annihilation rate of an atom or molecule normalised to a free electron gas of the same density and is given by:

$$
Z_{e f f}=\frac{\Gamma}{\pi r_{0}^{2} c n_{m}},
$$

where $\Gamma$ is the annihilation rate, $r_{0}$ is the classical radius of the electron (positron), $\mathrm{c}$ is the speed of light and $\mathrm{n}_{\mathrm{m}}$ is the target number density. In the absence of any correlations, $Z_{\text {eff }}$ should be of the same order as the number of electrons on the target. However, in the case of hydrocarbon and substituted hydrocarbon molecules, it was found that $\mathrm{Z}_{\text {eff, }}$ using room temperature positrons, could be many orders of magnitudes larger than $Z$, with the difference increasing rapidly with the size of the molecule [12]. Reasons postulated to explain this discrepancy mainly centred on the existence of positron-molecule resonances or bound states [13], where the incident positron is bound to the target molecule (temporarily in the case of a resonance), thus increasing the time spent in the vicinity of the target electrons and therefore the annihilation rate. The nature of the possible resonances or bound states has remained a mystery until recently due to a lack of experimental measurements capable of testing any of the theories.

Using the technique outlined above, the first measurements of annihilation as a function of incident

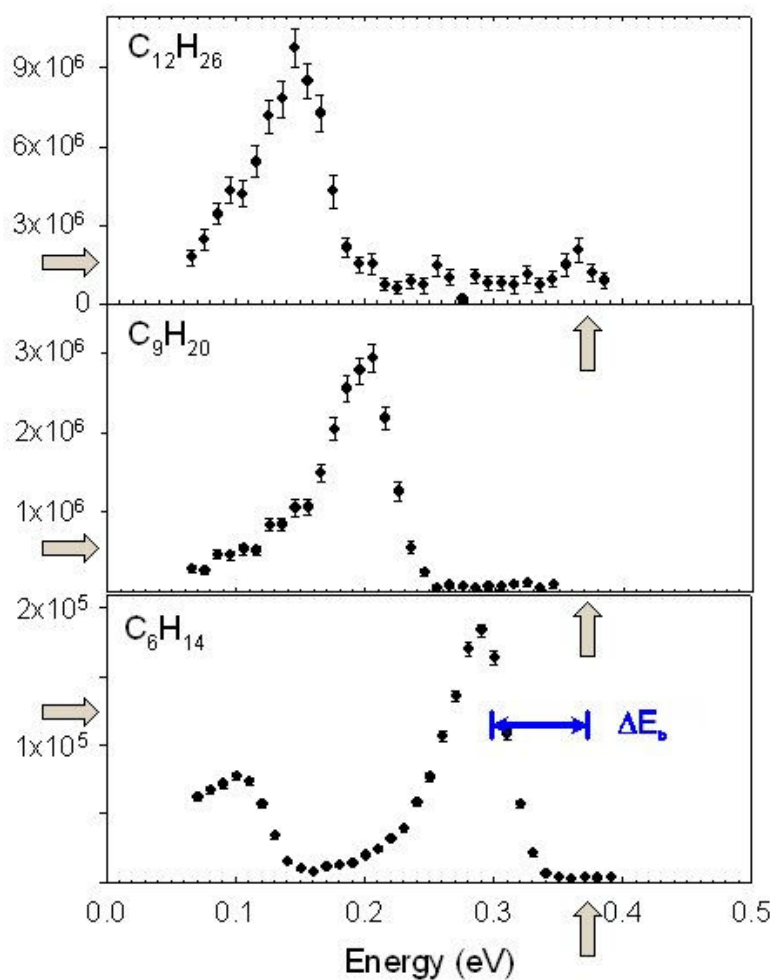

Figure 4: $Z_{\text {eff }}$ (see text) as a function of energy for dodecane, nonane and hexane.

The arrow on the horizontal axis marks the position of the $\mathrm{C}-\mathrm{H}$ stretch vibrational mode.

The binding energy for hexane is shown. energy were made for a wide variety of target molecules [11]. Some of the results are shown in Fig. 4. It can be seen that there are large peaks in the spectra, that correspond to the existence of vibrational modes of the molecules. In particular, the largest peak in each spectrum corresponds to the $\mathrm{C}-\mathrm{H}$ stretch mode of the molecular vibrations. The peaks in the annihilation spectra are shifted downwards in energy relative to the vibrational modes of the molecules. This can be interpreted in the framework of a recent theory that predicts the

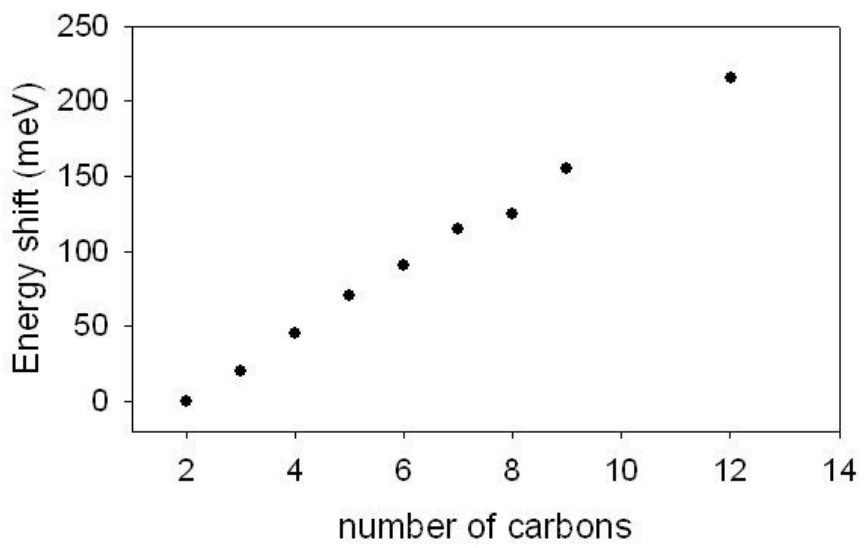

Figure 5: Binding energy as a function of the number of carbon atoms for the alkanes $\left(\mathrm{C}_{n} \mathrm{H}_{2 n+2}\right)$. 
existence of a positron bound state which then supports vibrational resonances in the positronmolecule complex [14]. The shift relative to the vibrational modes of the neutral target is a measure of the positron binding energy. It can be seen that in the case of hydrocarbon chains, this binding energy increases with the number of carbon atoms in the molecule. Fig. 5 shows the binding for alkanes with up to 12 carbons.

Excitation, Ionisation and Positronium Formation. By exploiting the behaviour of the positron beam in a strong magnetic field, it is possible to study other scattering processes. The energy of each positron in the beam can be divided into two components, so that $E_{T}=E_{\|}+E_{\perp}$, where $E_{T}$ is the total energy, $\mathrm{E}_{\|}$is the energy in the direction parallel to the field and $\mathrm{E}_{\perp}$ is the energy component in the cyclotron motion of the positron, perpendicular to the field. If a positron passes through a gas cell and is scattered (as shown in Fig. 2), then the energy will be redistributed. Scattering through an angle transfers energy from $\mathrm{E}_{\|}$to $\mathrm{E}_{\perp}$, and any energy loss process will reduce the total energy. The energy spread of the positron beam can be analysed using a retarding potential analyser (RPA), which is sensitive only to $E_{\|}$. If the RPA is located in a magnetic field of the same magnitude as the scattering cell, the distribution of $\mathrm{E}_{\|}$will give information about the differential scattering. This idea has been used to make measurements of low energy differential elastic scattering for noble gases.

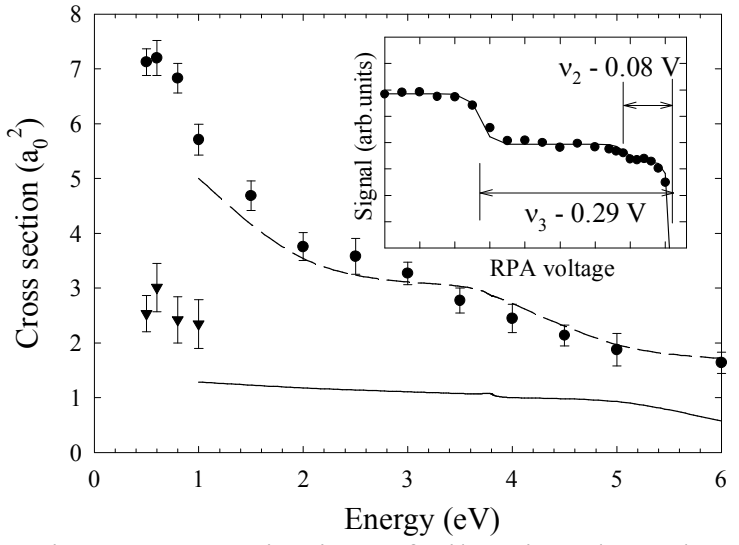

Figure 6: Excitation of vibrational modes of $\mathrm{CO}_{2}$ by positron impact, showing a comparison to theory [16]. Circles are the $v_{3}$ mode and triangles are the $v_{2}$ mode. The insert shows the energy loss steps observed using the RPA.
If the RPA is located in a field smaller than that of the scattering cell, then the adiabatic invariance of the quantity $\mathrm{E}_{\perp} / \mathrm{B}$ means that $\mathrm{E}_{\perp}$ will be converted back into $\mathrm{E}_{\|}$by an amount depending on the ratio of the field strengths. In this case, angular scattering information is lost, but with a sufficiently high field ratio (35 is used currently) the proportion of the positron beam that has lost total energy can be measured. This technique can be used to measure excitation and ionisation cross sections. An example of the first state selective vibrational excitation cross sections for positron impact is shown in Fig. 6, in this case for the excitation of the asymmetric stretch $\left(v_{3}\right)$ and the bending mode $\left(v_{2}\right)$ of $\mathrm{CO}_{2}$. This work has also been extended to include measurements of electronic excitation and ionisation cross sections [9]. In addition to being a first for positron measurements, this technique compares very favourably to conventional techniques for the measurement of total excitation cross section by electron impact [1].

If the energy of the positrons is such that positronium formation is possible (E>IP-6.8 eV), then the loss of positrons from the beam, and thus the positronium formation cross section, can be measured. A full discussion of scattering in a magnetic field and its application can be found in Ref. [8].

\section{Future Directions}

It is anticipated that the previous techniques, as well as some new approaches can be used to study the properties of clusters and large molecules. For instance, $\mathrm{C}_{60}$ is predicted to exhibit a novel "cage-state" resonance with the positron trapped inside the molecule [17]. This can potentially be a sensitive probe of the interior of this system. A potentially significant application of positrons as a probe of clusters is positron-induced Auger spectroscopy. In this case, a positron annihilates with an inner shell electron of an atom in the cluster, resulting in an Auger decay where a secondary 
electron is emitted. Analysis of this Auger electron can then provide surface-specific information, as low energy positrons will not penetrate deep into the cluster. This has large advantages over electron-induced Auger decay, where the incident electrons have relatively high energy to knock out an inner shell electron from the target, and there is a resultant large background from the many unwanted secondary electrons. Various other techniques can also be employed in the study of clusters and large molecules, such as positron induced ionisation and positron attachment.

\section{Conclusion}

The new technology of buffer gas positron traps has made available a new generation of highresolution low-energy positron beams. They are versatile tools for the study of positron scattering processes and have enabled a variety of new scattering measurements. These include the first observation of positron-molecule resonances, evidence for positron-molecule bound states and measurements of state selective excitation processes. Using the techniques developed here, scattering with both electrons and positrons can probe the electronic and vibrational states and size of clusters, in situ, in vacuo. Both neutral clusters and cluster ions can be studied in detail, the latter in a trap with a several-tesla magnetic field.

\section{Acknowledgements}

This work was supported by the U.S. National Science Foundation and Office of Naval Research.

\section{References}

[1] S. J. Buckman, in New Directions in Antimatter Chemistry and Physics, edited by C. M. Surko and F. A. Ginaturco (Kluwer Academic Publishers, Dordrecht, 2001) p. 391.

[2] Positron Beams and their Applications, edited by P. Coleman (World Scientific, Singapore, 2000)

[3] for example, S. Mori and O. Sueoka, J. Phys. B 27, 4349 (1994), K. Paludan et al., J. Phys. B 30, L581 (1997) and references therein.

[4] for example, T.S. Stein et al., Hyperfine Interact. 73, 53 (1992), L.S. Fornari et al., Phys. Rev. Lett. 51, 2276 (1983), G. Laricchia et al., J. Phys. B 352525 (2002) and references therein.

[5] for example W.E. Kauppila and T.S. Stein, Adv. At., Mol., Opt. Phys. 26, 1 (1990), M. Kimura et al., Adv. Chem. Phys. 111, 537 (2000) and references therein.

[6] T.J. Murphy and C.M. Surko, Phys. Rev. A 46, 5696 (1992)

[7] S.J. Gilbert et al., Appl. Phys. Lett. 70, 1944 (1997)

[8] J. P. Sullivan et al., Phys. Rev. A 66, 042708 (2002)

[9] S.J. Gilbert et al., Phys. Rev. Lett. 82, 5032 (1999), J.P. Sullivan et al. Phys. Rev. Lett. 86, 1494

(2001), J.P. Sullivan et al., Phys. Rev. Lett. 87, 073201 (2001), S. J. Gilbert et al., Phys. Rev. Lett. 88, 043201 (2002)

[10] C.M. Surko et al., in Non-Neutral Plasma Physics III, edited by J.J. Bollinger, R.L. Spencer, and R.C. Davidson (AIP, New York, 1999), pp. 3-12.

[11] S. J. Gilbert et al., Phys. Rev. Lett. 88, 043201 (2002), L. D. Barnes et al., Phys. Rev. A 67, 032706 (2003)

[12] K. Iwata et al., Phys. Rev. A 61, 022719 (2000) and references therein

[13] for example, P. M. Smith and D. A. L. Paul, Can. J. Phys. 48, 2984 (1970), C. M. Surko et al., Phys. Rev. Lett. 61, 1831 (1988), G. Laricchia and C. Wilkin, Phys. Rev. Lett. 79, 2241 (1997), G.

F. Gribakin, Phys. Rev. A 61, 022720 (2000) and references therein

[14] G. F. Gribakin, Phys. Rev. A 61, 022720 (2000)

[15] S.J. Gilbert et al., Phys. Rev. Lett. 82, 5032 (1999)

[16] M. Kimura et al., Phys. Rev. Lett. 80, 3936 (1998)

[17] Gianturco and Lucchese, Phys. Rev. A 60, 4567 (1999) 